\title{
Evaluating Open Collaboration Opportunities in the Fire Service with FireCrowd
}

\author{
Eleanor R. Burgess \\ University College London \\ eleanor.burgess.15@ucl.ac.uk
}

\author{
Aaron Shaw \\ Northwestern University \\ aaronshaw@northwestern.edu
}

\begin{abstract}
In emergency response organizations like the fire service, personnel require easy access to reliable, up-to-date safety protocols. Systems for creating and managing Standard Operating Procedures (SOPs) within these command and control organizations are often rigid, inaccessible, and siloed. Open collaboration systems like wikis and social computing tools have the potential to address these limitations, but have not been analyzed for intra-organizational use in emergency services. In response to a request from the Fire Protection Research Foundation (FPRF) we evaluated a high-fidelity open collaboration system prototype, FireCrowd, that was designed to manage SOPs within the U.S. fire service. We use the prototype as a technology probe and apply human-centered design methods in a suburban fire department in the Chicago area. We find that organizational factors would inhibit the adoption of some open collaboration practices and identify points in current practices that offer opportunities for open collaboration in the future. ${ }^{1}$
\end{abstract}

\section{Keywords}

Organizations; emergency response; open collaboration; knowledge management

\section{Categories and Subject Descriptors}

H.5.m. [Information Interfaces and Presentation (e.g. HCI)]: Miscellaneous

\section{INTRODUCTION}

We investigate whether open collaboration systems can enhance knowledge management around safety protocols within the U.S. fire service. Fire fighters and emergency response personnel need fast, reliable access to up-to-date safety protocols in the field. However, in the fire service, Standard Operating Procedures (SOPs) are written and approved exclusively within individual departments

\footnotetext{
${ }^{1} \mathrm{~A}$ version of this work licensed under a Creative-Commons Attribution International 4.0 License is available from one author's personal website or by contacting the authors.

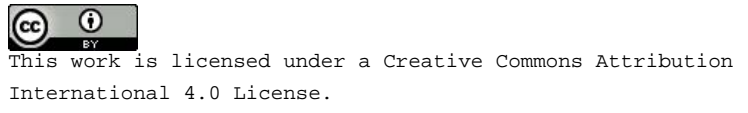

OpenSym '16 August 17-19, 2016, Berlin, Germany

(C) 2016 Copyright held by the owner/author(s).

ACM ISBN 978-1-4503-4451-7/16/08.

DOI: http: //dx.doi .org/10.1145/2957792 .2957794

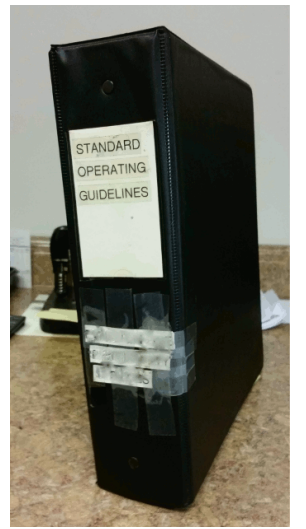

Figure 1: The SOP binder in the fire department where we conducted this study.

even though many departments confront similar situations. Protocols are infrequently updated and are usually stored in paper files or desktop computers that never leave the firehouse (see Figure 1). These challenges pose an urgent threat to the health and safety of fire service personnel.

Open collaboration systems such as wikis or social Q\&A platforms support collaborative, distributed knowledge management [6]. Prior work has shown how open collaboration systems support cooperative work in firms $[2,12]$ and other settings, but has not evaluated these systems within emergency response contexts. Earlier studies of collaboration technologies in emergency response emphasize how cultural tensions between different organizations (e.g., fire service, police, and emergency medical services) prevent effective cooperation [9]. In contrast, we explore the opportunities for open collaboration in a critical knowledge management system within a single branch of emergency response: the fire service.

At the invitation of the FPRF, we evaluated FireCrowd, a prototype open collaboration system for SOP creation and revision in the U.S. fire service. ${ }^{2}$ We used the FireCrowd prototype as a technology probe, applying qualitative, human-centered design methods to assess opportunities for open collaboration in a single fire department. We extend prior HCI research on technology adoption in organizations and open collaboration systems by focusing on a novel domain: knowledge management within a command and control emergency response organization. Our findings confirm the potential of open collaboration systems to overcome knowledge management inefficiencies, but underscore how organizational structures and routines would also inhibit collaboration and openness.

\footnotetext{
${ }^{2}$ See http://firecrowd.com
} 


\section{PRIOR WORK \& CONTEXT}

Open collaboration and social computing systems offer unique advantages for facilitating knowledge sharing, transactive memory, coordination, and information diffusion across a number of domains $[2,6,12]$. However, introducing open collaboration into the safety protocol management of a command and control organization such as the fire service presents specific design challenges. On the one hand, systematic SOP review is necessary to ensure high quality, reliable protocols. On the other, protocols should be updated quickly and take advantage of the knowledge spread across individuals in many departments. These competing demands create tensions between hierarchy and openness that existing research has not explored.

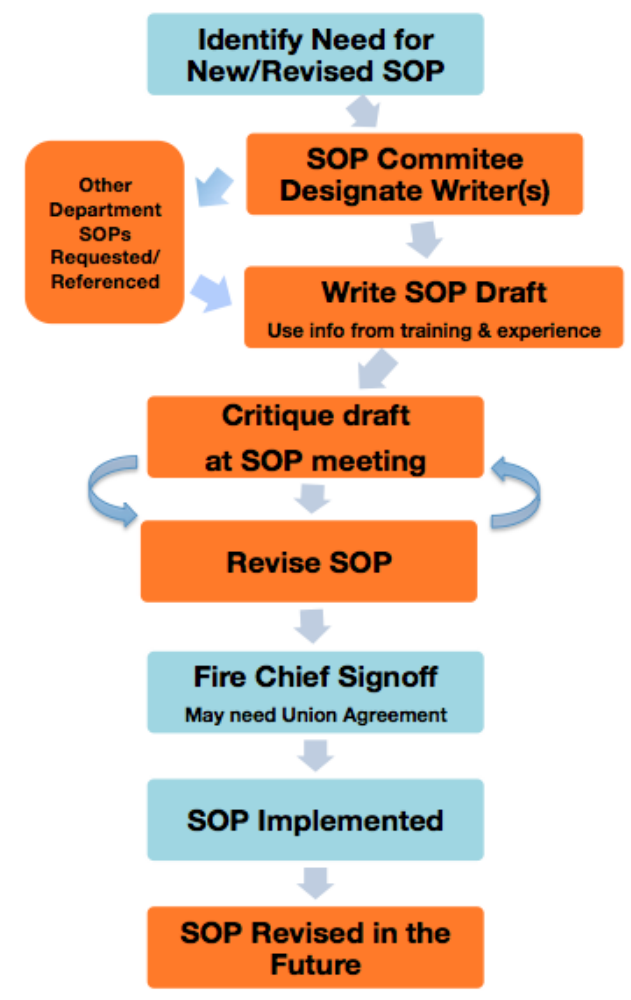

Figure 2: Diagram of a typical fire department SOP creation process. The FPRF designed FireCrowd to intervene in the steps represented in orange boxes.

HCI scholars have analyzed systems to support the operations of public safety organizations with command and control structures, including fire and police departments $[4,10,9]$. Some have examined the adoption of mobile computing systems in the fire service (e.g., [10]) and intelligence communities [1,3]. Recent studies investigate organizational uses of social media systems in the context of crisis response [7, 13]. This prior work demonstrates how organizational factors such as boundary management, professional closure, norms, and organizational structure can inhibit information sharing and collaboration. A related study by Ley and colleagues [9] shows that technological systems that support context awareness can facilitate coordination and communication across emergency response organizations. We are not aware of studies examining opportunities for open collaboration systems within command and control organizations where poor knowledge management threatens the lives and safety of system users.

\subsection{Standard Operating Procedures}

We focus on a critical intra-organizational knowledge management system in the United States fire service: Standard Operating Procedures. SOPs are prescriptive protocols that fire fighters follow when responding to calls. The protocols vary widely and cover topics from high-rise building fires to proper hose operations. Within the United States, fire fighters are trained using SOPs and held accountable to them on the job. In order to ensure SOP quality, departments typically follow a multi-step process to draft, review, authorize, implement, and revise their procedures (see Figure 2). Many fire fighters provide writing and editing input and the chief provides final signoff before the SOP is put into action.

Fire service SOP creation and revision routines lack optimal mechanisms to incorporate new knowledge quickly, leading to avoidable safety risks [5]. A striking example of this occurred when electric and hybrid vehicles first became popular. During incidents involving the near-silent motors of these vehicles, fire fighters attempted to extinguish fires without realizing that the cars were still running and unoccupied, burning cars would start driving away [5]. It took a long time for this knowledge to diffuse into individual fire department's SOPs.

These limitations around SOP creation and management led the FPRF, a research body serving the National Fire Protection Association, to approach a member of our research team about evaluating an open online collaboration system to manage SOPs at a national level within the fire service. The foundation funded the creation of the prototype, which we evaluated in the current study. Below we briefly describe the prototype, research methods, and setting for our evaluation.

\subsection{The FireCrowd Prototype}

FireCrowd is a high-fidelity prototype open collaboration system. The prototype was created under the direction of an FPRF advisory board of fire service experts. It uses a customized Drupal template that allows collaborative editing, manages user roles and authentication, and facilitates the aggregation of collaborativelyauthored documents (see Figure 3).

FireCrowd enables U.S. fire service professionals to collaboratively author, share, and access SOPs at a national level. The prototype assumes users are integrated into an existing department SOP workflow and introduces open collaboration at strategic points (see Figure 2). Following FPRF specifications, the system presents users with a repository of editable SOPs in-progress. Changes to the SOPs in-progress are reviewed on a weekly basis by a fire fighter safety expert, who then implements approved changes in a parallel repository of vetted SOPs. Vetted SOPs are made available for download and further department-specific revision.

\section{METHODS}

We apply qualitative, human-centered design methods to analyze current fire service practices around SOPs and the viability of the FireCrowd prototype. Using the FireCrowd prototype as a technology probe [8], we explore opportunities for open collaboration and knowledge management in the fire service through usability tests, contextual inquiries, and a large focus group. Presenting fire fighters with the FPRF's prototype system elicited direct feedback and responses in a way that interviewing or observations alone (without the prototype) would not have allowed.

Our empirical work took place through interviews with fire service safety experts and in a fire department we partnered with for the purpose of the study. Our partner fire department serves a suburban, primarily residential community of about 75,000 people over 


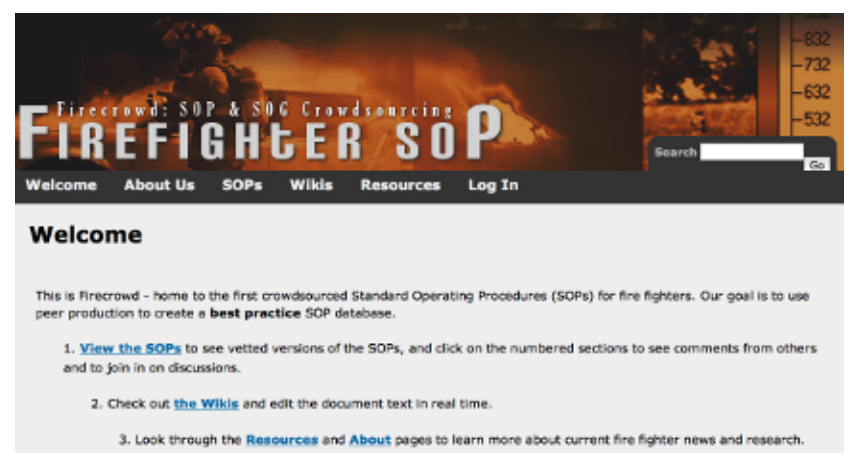

Figure 3: FireCrowd Landing Page

7.8 square miles in the Chicago area. The department maintains a full-time staff of 110 members at five stations.

We conducted usability tests of the FireCrowd prototype with three fire fighter participants each working at a different fire station within our partner department. These tests were completed using fire station desktop computers. Following a study protocol which approximated a typical user set of site actions, we prompted participants to complete example tasks using FireCrowd, (e.g. "log in, find $[X]$ SOP, edit $[X]$ SOP, etc."). We then gathered design feedback and contextual information about technology use in the fire house environment. We audio recorded these usability tests and took written field notes.

In addition, we conducted contextual inquiries with two fire safety chiefs; one with the Division Chief of Operations and Training at our partner department, and one with the safety chief of an East Coast department well-regarded for their quality SOPs. We took written notes regarding their department processes of new SOP development, implementation, and revision. We also held followup phone conversations to clarify details in response to topics that emerged as we analyzed their original responses.

Finally, we facilitated a focus group of the 33 SOP Committee members in our partner department. We presented FireCrowd to the group and then solicited feedback about SOP procedures in writing and discussion following a study protocol (available upon request). We collected all written responses, audio recorded the meeting, and took detailed written notes.

Data collection took place over a 12 week period in 2014, yielding field notes, interview recordings and transcripts, photographs, and fire fighters' written responses to focus group questions. We reviewed, summarized, and analyzed all of these materials. We developed inductive codes based on prior literature and emergent themes. Then, over three rounds of analysis, we elaborated the codes in written memos, refined them, and, re-applied the refined codes to the study documents and notes.

\section{FINDINGS}

Our observations confirm that SOPs are important political and social artifacts that organize work in the fire service. Probing the existing SOP system with FireCrowd, we find that fire fighters perceive open collaboration as a threat to the norms of SOP creation and implementation. However, we also find opportunities for open collaboration to solve urgent knowledge management challenges.

\subsection{Open Collaboration as a Threat}

The routines and command hierarchy of the fire service shape fire fighter attitudes toward the control of SOPs. Because the creation and validation of SOPs traditionally reside at the level of each fire department, fire fighters express a strong sense of ownership over the content of "their" SOPs. However, in the FireCrowd prototype, individuals from many departments are encouraged to edit SOP content. This provokes apprehension among would-be users. For example, several members the fire fighter focus group opposed the idea of outsiders changing department work. One standard-rank SOP Committee fire fighter articulated his concern:

That's almost a deal-breaker for us. Having a third party be able to go through our process and our SOPs...the reason we have this whole [SOP] Committee is that our process is for our department based on our needs and makes us unique.

Department-level identity, boundaries, and SOP content ownership would inhibit collaboration.

Departments also use SOPs as tools to describe expectations and to evaluate fire fighter job performance. The degree of individual fire fighter accountability to the SOP often depends on their Batallion Chief. One department safety chief argued for strict adherence to SOPs: "Fire fighters should 'stay in the box' and use [the SOPs] they learned in training. If a fire fighter does deviate with good reason, fine, but you have to have a good reason." In such a case, the SOPs express departmental norms directly and are not suitable for editing across department boundaries. More open collaboration threatens to undermine department-level hierarchy and rules.

\subsection{Open Collaboration as Opportunity}

Conversations with fire fighters and chiefs also revealed several failure points in existing departmental knowledge management routines that open collaboration systems can ameliorate. These failure points include the length and constraints of the SOP creation process as well as general problems with SOP accessibility.

Fire fighters and chiefs perceive SOP creation as time consuming, slow, and inefficient. The current process for our partner department looks like this: one or two fire fighters write a new SOP on their own (unpaid) time. Then the document is edited by the SOP Committee, often going through several rounds of review before final signoff from the chief. Fire fighters must be trained on the new SOP, which takes additional time because, as the department safety chief said, "firemen don't accept change very well." As a result of these delays, SOPs are often poorly maintained and, in the words of one focus group participant, "reactive instead of proactive." Department members recognize the need to revise old SOPs, but fail to do so in a timely manner because of the difficult process. The safety chief at our partner department reiterated that relying on outdated SOPs introduces unnecessary risks.

Our study participants felt that open, collaborative editing would facilitate faster rounds of SOP drafting and revision, better version control, and easier review. The focus group became excited about the ability to support rapid, collaborative intra-departmental edits to SOPs without waiting for the next committee meeting. Another focus group participant spoke about specific collaborative editing features that he would find useful: "When I make changes to an old [SOP], they are hard for other people to identify...Using color...anything added or removed is easy to see." Basic collaborative text editing functions such as tracked changes and version control would address this need.

Respondents also underscored that fire fighters struggle to access and stay up-to-date on SOPs. In our partner department, fire fighters access SOPs in three ways: a large SOP binder in each station (see Figure 1); an intranet SOP database on fire house computers; or, in some cases, a small computer mounted in the fire truck (rig). 
All of these methods have limitations identified by the fire fighters, including poor searchability, mobility, and version control.

Study participants saw online and mobile access to SOPs as potential solutions to these problems. One standard-level fireman spoke about burning mailbox calls that are rare, but require a special procedure. He suggested that a smartphone interface could be used to check the SOP in the field. Another recommended that cross-references and suggested searches would be valuable.

\section{DISCUSSION}

Our findings illustrate how tensions between openness and hierarchy play out in the context of fire service knowledge management. Fire service personnel want higher-tempo forms of collaboration and openness to overcome limitations of existing knowledge management infrastructure. They perceive several ways open collaboration systems might help achieve these goals. However, they also make it clear that some technical affordances of open collaboration contradict key organizational practices. They reject forms of openness that undermine their department command structure.

These findings suggest that open collaboration platforms for SOP management need to be designed and adapted to suit the organizational setting and structure of the fire service more than the FPRF anticipated. For example, any open collaboration system adopted in such a setting needs to afford accountability to versions of the text that correspond to the specific needs, norms, and routines of individual departments. In many cases, fire fighter concerns could be addressed by providing open and flexible access to the SOPs rather than fundamentally changing how SOPs are created. Additional prototyping and evaluation in a larger, more diverse sample of departments can test whether these observations generalize.

The results extend prior literature by probing open collaboration opportunities within the fire service, a command and control public safety organization. Whereas prior work emphasized the advantages of open collaboration for knowledge management, the FireCrowd prototype reveals how open collaboration systems contradict fire service routines and structures. These contradictions affect both fire fighter understandings of such systems as well as their attitudes toward system adoption. Our work confirms that organizational environments shape technology adoption.

\section{CONCLUSION}

The current study investigates open collaboration and knowledge management within command and control organizations where information reliability and safety are critical concerns. Many emergency response organizations utilize SOPs and the findings we report may be applicable to other contexts including law enforcement. Future research should expand this important area of open collaboration design and evaluation.

\section{ACKNOWLEDGEMENTS}

Thanks to our partner fire department, Casey Grant and the FPRF, Madhu Reddy, Darren Gergle, Anne Marie Piper, Haoqi Zhang, Liz Gerber, and others for their support and help with this project.

\section{REFERENCES}

[1] D. C. Andrus. Toward a Complex Adaptive Intelligence Community: The Wiki and the Blog. Studies in Intelligence, 49(3):2005-2006, Apr. 2007.

[2] O. Arazy, I. Gellatly, S. Jang, and R. Patterson. Wiki deployment in corporate settings. IEEE Technology and Society Magazine, 28(2):57-64, 2009.

[3] R. A. Best Jr. Intelligence Information: Need-To-Know Vs. Need-to-Share. Congressional Research Service. Diane Publishing, Darby, PA, July 2011.

[4] L. Carver and M. Turoff. Human-computer Interaction: The Human and Computer As a Team in Emergency Management Information Systems. Commun. ACM, 50(3):33-38, Mar. 2007.

[5] J. D. Emery. Hybrid Vehicles: Separating Fact from Fiction. Fire Engineering, 162(7):73-82, July 2009.

[6] A. Forte and C. Lampe. Defining, Understanding, and Supporting Open Collaboration Lessons From the Literature. American Behavioral Scientist, 57(5):535-547, May 2013.

[7] A. L. Hughes, L. A. A. St. Denis, L. Palen, and K. M. Anderson. Online Public Communications by Police and Fire Services During the 2012 Hurricane Sandy. In Proceedings of the SIGCHI Conference on Human Factors in Computing Systems, CHI '14. ACM, 2014.

[8] H. Hutchinson, W. Mackay, B. Westerlund, B. B. Bederson, A. Druin, C. Plaisant, M. Beaudouin-Lafon, S. Conversy, H. Evans, H. Hansen, N. Roussel, and B. Eiderbäck. Technology Probes: Inspiring Design for and with Families. In Proceedings of the SIGCHI Conference on Human Factors in Computing Systems, CHI '03. ACM, 2003.

[9] B. Ley, V. Pipek, C. Reuter, and T. Wiedenhoefer. Supporting Improvisation Work in Inter-organizational Crisis Management. In Proceedings of the SIGCHI Conference on Human Factors in Computing Systems, CHI '12. ACM, 2012.

[10] Á. Monares, S. F. Ochoa, J. A. Pino, V. Herskovic, J. Rodriguez-Covili, and A. Neyem. Mobile computing in urban emergency situations: Improving the support to firefighters in the field. Expert Systems with Applications, 38(2):1255-1267, Feb. 2011.

[11] W. J. Orlikowski. Learning from Notes: Organizational Issues in Groupware Implementation. In Proceedings of the 1992 ACM Conference on Computer-supported Cooperative Work, CSCW '92. ACM, 1992.

[12] D. Riehle, J. Ellenberger, T. Menahem, B. Mikhailovski, Y. Natchetoi, B. Naveh, and T. Odenwald. Open Collaboration within Corporations Using Software Forges. IEEE Software, 26(2):52-58, Mar. 2009.

[13] A. H. Tapia and K. Moore. Good Enough is Good Enough: Overcoming Disaster Response Organizations' Slow Social Media Data Adoption. Computer Supported Cooperative Work (CSCW), 23(4-6):483-512, July 2014. 